Guillermo Oscar Quinteros,

Cuadernos de H Ideas, vol. 11, n. 11, e001, diciembre 2017

ISSN 2313-9048 | https://doi.org/10.24215/23139048e001

http://perio.unlp.edu.ar/ojs/index.php/cps/index

FPyCS | Universidad Nacional de La Plata

La Plata | Buenos Aires | Argentina

\title{
A cien años de la Reforma Universitaria: Entrevista a Raúl A. Pessacq, un reformista para construir la Universidad de la democracia.
}

\section{One hundred years of University Reform: Interview with Raúl A. Pessacq, a reformer to build the University of democracy.}

\author{
Guillermo O. Quinteros \\ Universidad Nacional de La Plata, Argentina. \\ goscarquinteros@gmail.com
}

Esta entrevista se realizó como una actividad paralela a una investigación mayor que llevamos adelante con el Dr. Guillermo Banzato y con el propio entrevistado. La publicamos en las vísperas del aniversario número 100 de La Reforma Universitaria, comenzada en Córdoba con una proclama estudiantil (el denominado "Manifiesto Liminar") escrita y leída por Deodoro Roca el 21 de junio de 1918.(1) Al margen de los avatares sufridos por la sociedad argentina en general y por la Universidad en particular, resulta de importancia recordar que en el año 1983 se puso fin al peor de los períodos de dictadura vividos por toda la población. Muchos varones y mujeres se comprometieron en una nueva etapa para desarmar los nefastos entramados autoritarios de la Dictadura militar. Uno de ellos fue el Ingeniero Raúl Adolfo Pessacq. Comprometido con la construcción de la democracia asumió el desafío de generar un marco legal para poner en funcionamiento una nueva Universidad Pública y al mismo tiempo, llevarlo a la práctica en la Universidad Nacional de La Plata, en la que fue su Rector Normalizador (1983-1986). 
Raúl Adolfo Pessacq, conocido por todos como "Otto", nació en la ciudad de La Plata el 17 de noviembre de 1941. Obtuvo el título de Ingeniero Químico en 1965 después de los cinco años de cursada de dicha carrea, regularidad que demuestra una importante dedicación al estudio. Pero su actividad como estudiante no se agotó en el ello, puesto que Otto, desarrolló una militancia muy activa como miembro de la agrupación ALU (Agrupación Liberal Universitaria).(2) Entre 1960 y 1965 fue Secretario y Presidente del Centro de Estudiantes de Ingeniería, Consejero Superior y presidente de la Federación Universitaria de La Plata. Pasó por todos los cargos docentes, desde Ayudante Alumno hasta Profesor Titular. En 1972 ingresó al CONICET y llegó hasta el cargo de Profesional Principal.

Guillermo O. Quinteros (GOQ): ¿Cómo fue que se involucró en el proceso de normalización de la UNLP?(3)

Raúl A. Pessacq (RAP): Fue la consecuencia de la actividad que venía realizando desde unos años antes en la Fundación Eugenio A. Blanco de La Plata.

En 1979 conocí a Raúl Alfonsín, quedé impresionado por sus ideas y su personalidad, y desde ese momento retomé la actividad política en la Unión Cívica Radical. Estaba afiliado desde 1972.

Con varios amigos de la época estudiantil reformista de los años sesenta, y otros radicales más jóvenes, creamos la Fundación Eugenio A. Blanco de La Plata en 1980, y comenzamos a realizar reuniones, dar charlas y a realizar proyectos políticos. Los presidentes honorarios eran el Dr. Carlos R. S. Alconada Aramburú y los Ingenieros Juan Sábato y Andrés Ringuelet.(4)

En la Fundación redactamos el primer proyecto de recuperación de las Universidades Nacionales, y a partir de 1982 lo presentamos en encuentros y reuniones sobre educación. Interactuamos con el Centro de Participación Política (CPP), que estaba formado por docentes, investigadores y mujeres y hombres de la cultura.(5)

Recuerdo que me tocó exponer sobre el tema en una mesa redonda con René Barbich, Fredi Storani y Andrés Ringuelet, que se realizó en el anfiteatro de física, en el año 1982.(6) Ya en octubre de 1983, di una charla para miembros de la UCR y de la Fundación, sobre las leyes universitarias, las intervenciones y las transiciones entre gobiernos civiles y golpes militares. La consecuencia de estas tareas fue que terminara involucrándome con la normalización de las universidades. 
GOQ: Pero usted ¿no venía trabajando en política universitaria desde antes de los $80 ?$

RAP: Si, desde que era estudiante. Mientras estudiaba, entre 1960 y 1965, fui dos veces presidente del Centro de Estudiantes de Ingeniería, presidente de la FULP,(7) consejero superior y asambleísta universitario. Participé en la elección de dos presidentes: Carlos S. Bianchi y Roberto Ciafardo. Una vez egresado, me fui a trabajar a la actividad privada.

Cuando volví a la Universidad en 1973, integré el grupo Línea con varios amigos reformistas y radicales. Redactamos varios proyectos técnicos y un anteproyecto de ley universitaria, con Héctor Rodolfo Demo y Juan Carlos Delorenzo, y lo presentamos ante varios diputados nacionales.(8) También fui uno de los propulsores de la Unión de Docentes e Investigadores Reformistas. En UDIR redactamos y publicamos en junio de 1974, una solicitada firmada por más de doscientos docentes e investigadores, solicitándole a las autoridades universitarias de ese momento (que respondían a la denominada Tendencia Peronista), la aplicación de la ley Taiana recién promulgada, la realización de concursos y otras cuestiones universitarias y políticas generales.(9) Fuimos violentamente rechazados y amenazados, razones por las que el grupo se disolvió.(10)

GOQ: Entonces ¿cortó con la militancia o siguió trabajando dentro de alguna agrupación o partido político?

RAP: No volví a tener actividad política hasta fines de los años setenta. Como te decía, luego de 1979 comencé a trabajar en la Fundación Eugenio A. Blanco de La Plata, y a asistir a reuniones de la UCR. Esta actividad se aceleró mucho a partir de la guerra de Malvinas.

GOQ: ¿Quién lo llamó para que se hiciera cargo de elaborar el texto del decreto 154/83? ¿Por qué usted, lo sabe, se lo dijeron?

RAP: El Dr. Carlos Alconada Aramburú me había comunicado, a principios de noviembre de 1983, que sería designado Rector de la Universidad. Solo unos días después me encargó la tarea de redactar el decreto de intervención de las universidades nacionales. Los ofrecimientos me sorprendieron.(11) El Dr. Alconada me conocía del trabajo en la Fundación, del estudio de las leyes universitarias, y de varias reuniones que habíamos tenido junto con otros integrantes con los que ya veníamos trabajando en temas universitarios, y de ciencia y técnica. Fueron esos trabajos dentro de la Fundación los que influyeron en la designación.(12) 
GOQ: Entonces, una vez que lo convocaron ¿cuál fue su actitud? Porque tenía en sus manos la potestad -por decirlo así- de diseñar a la Universidad pública. ¿Qué pensó en ese momento?

RAP: Por supuesto que no dudé en aceptar, aunque con más temor que el que había tenido solo unos días antes por la designación. Pero en ese momento estaba convencido que había que hacer lo que nos correspondiera y trabajar en el lugar que nos eligieran para terminar con la dictadura del Proceso. La cuestión seria comenzó cuando me puse a pensar en cómo redactar el borrador del decreto.

Lo escribí solo y sin consultar porque el tema era urgente y ya lo habíamos discutido varias veces en la Fundación. Creo que fue la prueba de que podría ser rector, y no tuve dudas una vez que logré decidirme, sobre lo que pensaba que había que hacer. Estaba muy convencido, sobre todo de las ideas que sustentaban el decreto, porque ya habían sido probadas años antes. Entonces le presenté el escrito al Dr. Alconada. Él corrigió los considerandos, pero no alteró el articulado. Luego lo complementó con el Decreto № 228.(13)

GOQ: Ahora..., para encarar toda esta tarea tuvo que ponerse a estudiar!

RAP: No..., más que estudiar! Tuve que pensar, consultar y repasar documentos, recordar todo lo que había vivido, leído y escrito durante los años anteriores, los de estudiante y los de docente, pero en especial los proyectos que habíamos escrito en la Fundación.

La base fueron los artículos sobre educación del libro de Alconada "La República Democrática, obra del Radicalismo", donde define a la autonomía como el verdadero y esencial carácter de la Universidad y brinda un marco jurídico y conceptual acerca de ella.(14)

La cuestión era cómo realizar la transición de la universidad de la dictadura a la universidad autónoma en el menor tiempo posible, porque para eso había que desarmar una estructura y fundar otra, que fuera la de la nueva democracia republicana.

GOQ: Pensó en la Reforma...

RAP: Así es, con la base de la Reforma Universitaria. Porque personalmente, entre 1960 y 1965, había estudiado en una universidad reformista que juzgo brillante. Además conocía su 
Estatuto porque fui Consejero Superior, o sea que había experimentado el funcionamiento de la Universidad reformista.

También había escrito un anteproyecto de ley, participado en la redacción de la solicitada de UDIR, y estudiado la legislación y las transiciones luego de las dictaduras y los golpes de estado. En esos períodos de inestabilidad política, las universidades siempre habían sufrido cesantías o expulsiones masivas de docentes e investigadores. Se dependía mucho de las decisiones personales, o sea arbitrarias de las autoridades y se padecía la discriminación ideológica.

Con este conocimiento y experiencia, pensé que había que evitar los defectos de toda esa historia universitaria muy negativa y retomar lo mejor del período de 1958 a 1966.

GOQ: ¿Cuáles fueron sus fuentes de información para darle un sentido político y administrativo a la Universidad?

RAP: El sentido político que se le dio a la intervención, a la que Alconada llamó normalización, era arribar con rapidez a la autonomía, con orden administrativo y -fundamentalmenteacadémico, con un claustro de profesores renovado y designado a partir de la realización de concursos por oposición y antecedentes. El sentido administrativo y académico estuvo dado por la adopción del estatuto como marco legal. El concepto global era la restitución de la república.

Las fuentes que se utilizaron para restablecerla fueron la ley Avellaneda, el decreto 6403/55, el estatuto de la UNLP de 1958, la solicitada de UDIR de 1974 y los trabajos que realizamos en la Fundación. El marco general fue la Plataforma de la UCR con la que había sido electo Raúl Alfonsín como presidente de la Nación, y que se había comprometido a cumplir.(15)

Solo teníamos treinta días para armar el gobierno y otra universidad, así que no teníamos tiempo y no podíamos ir más allá de lo que ya conocíamos y teníamos a mano, como fuentes de ideas.

GOQ: Cuando refiere a que en los 50 y 60 se vivió una época de esplendor de la Universidad pública, ¿lo dice sólo porque estaban en plena vigencia los principios de la Reforma? 
RAP: Sí, porque creo que la estructura universitaria de esos años, con plena autonomía, con cogobierno, con diversidad y pluralismo intelectual, permitía una gran libertad de pensamiento fomentando una importante formación cultural.

El claustro docente era excelente, y las razonables exigencias académicas permitían una muy buena formación profesional y científica.

Había existido un gran recambio de profesores a partir de 1955, se concursaron gran cantidad de cargos y los nuevos docentes eran relativamente jóvenes y propensos a los cambios y las innovaciones pedagógicas. Se crearon nuevas carreras, se revisaron los planes de estudio, se comenzaron nuevas investigaciones científicas y se estableció una relación importante con el CONICET y la CIC.(16) Se incrementaron las becas de perfeccionamiento en el exterior para la formación doctoral.

El funcionamiento de los Centros de estudiantes, de las Federaciones, de los Consejos Académicos y del Superior, y las elecciones de los claustros se realizaba con normalidad. Los decanos y rectores eran todas personalidades destacadas en sus profesiones.

Por entonces, ya se decía que la preparación de los colegios secundarios era insuficiente para ingresar a la universidad, y por eso es que en muchas carreras existían cursos de ingreso. Se cursaban materias anuales, se rendían parciales escritos y los exámenes finales eran orales y escritos, y teórico y prácticos. La exigencia de los docentes era importante. Se buscaba la excelencia en la formación de grado, con muy buena dedicación de los profesores. La recompensa del esfuerzo de los estudiantes, en esos años, era un título que garantizaba el progreso personal. Se decía que un título universitario era un seguro de vida. Todos teníamos proyectos y había muchas ofertas de trabajo en casi todas las profesiones (los destacados del entrevistador)

La década del sesenta fue de expansión, y de importantes cambios culturales, aún con los problemas políticos que tenía el país. Esa universidad que yo conocí, acompañaba el crecimiento y el progreso. Porque hay que recordar que existía pleno empleo y escasa pobreza.

La Reforma fue la condición necesaria para que esa Universidad fuera una brillante fuente de conocimiento, de investigación, de formación de profesionales, y que también promovía la formación de ciudadanos para la república.

GOQ: Ahora bien, ¿cuáles son para usted los principios reformistas que debían ser enfatizados? 
RAP: Todos los que conocíamos del funcionamiento de la Universidad reformista. Las ideas principales eran las contenidas en la plataforma política de la UCR en la que, por supuesto, estaban contemplados los principios reformistas tradicionales. El fundamento básico era la autonomía, razón por la cual se la debía restituir en la forma más rápida posible -solo un año con alguna extensión-, por lo que la intervención debía ser corta. De esa forma las universidades volverían a gobernarse por sí mismas, sin depender políticamente del poder ejecutivo.

El estatuto que garantizaba el funcionamiento dentro de un marco legal completo, era una importante condición inicial.(17)

Sostener la periodicidad de la cátedra y concursar la mayor cantidad de cargos docentes posibles, por oposición y antecedentes, con jurados idóneos y sin ningún tipo de discriminación, eran parte de ese marco legal. Pero también se debían revisar los concursos realizados por el denominado Proceso, porque muchos de ellos estaban viciados de nulidad por discriminación ideológica.

Restaurar el cogobierno en los consejos fue uno de principios que se retomó desde el inicio. Los estudiantes fueron designados por los centros y las federaciones que hubieran realizado elecciones, y los profesores y los graduados fueron incorporados poco tiempo después, a propuesta de los claustros. Esta intervención colegiada garantizaba el control de los actos administrativos y académicos del rector y de los decanos, además garantizaba su publicidad. Era la forma de evitar las discrecionalidades y las resoluciones personales.

Se debía actuar en forma pluralista con todas las tendencias y partidos políticos pero con independencia, aún de la UCR porque solo se recibían instrucciones del ministro.

Creo que, en definitiva, fue retomar en gran parte la estructura y el espíritu reformista de la universidad del 58 al 66, sin discriminaciones ni proscripciones políticas.(18)

GOQ: ¿Utilizó como guía a esos principios para elaborar el texto del Decreto? ¿En cuáles de sus artículos se manifestaron?

RAP: Por supuesto que fueron la guía! Se expresaron en todo el decreto! En el artículo 4e se ponen en vigencia los estatutos universitarios vigentes al 29 de julio de 1966. Esa era la forma de intervenir con una norma legal que ya había probado su excelencia. El Dr. Alconada, como jurista y republicano, no quiso derogar la ley del proceso, la № 22207 por decreto, por lo que hubo que atenerse a algunos de sus artículos. 
En los artículos $5^{\circ}$ y $6^{\circ}$ se constituyen los consejos superior y académicos. La intervención colegiada garantizaba que las autoridades fueran controladas, tal como se planteaba con la limitación de las acciones de los interventores en los Considerandos del decreto.

En el artículo $7^{\circ}$ y $8^{\circ}$ con la suspensión y revisión de los concursos en los que existían clausulas proscriptivas y discriminatorias. También el artículo $9^{\circ}$ con el reconocimiento de los centros de estudiantes, las federaciones regionales y la FUA, sobre la base del concepto de las centrales gremiales únicas. Se eliminaban las clausulas proscriptivas y discriminatorias en la universidad mediante el $10^{\circ}$, consagrándose la pluralidad.

El objetivo de la restauración de la autonomía estaba explícitamente citado en los Considerandos. La pluralidad política y el respeto por todas las expresiones políticas y culturales estuvieron garantizados por la forma de actuar del ministro, y su aceptación por los rectores y los decanos.

En definitiva eran los simples conceptos reformistas de los estatutos vigentes hasta 1966, que son complementarios de preceptos democráticos y republicanos de la Constitución Nacional.

GOQ: En el momento de elaborar la propuesta, ¿pensó en la UNLP o en todas las Universidades?

RAP: Pensé en todas las universidades nacionales, lo que se refleja en los artículos $4^{\circ}, 5^{\circ}$ y $6^{\circ}$ los que les daba libertad de elección de los estatutos y de la conformación de los consejos.

GOQ: ¿Recibió alguna instrucción sobre lo que debía hacer, sobre lo que debía dejar y sobre lo que debía cambiar? Lo ayudaron, tuvo colaboradores en ese proceso constructivo?

RAP: El ministro Alconada me dio algunas instrucciones y consejos porque solo había que ajustarse al texto del decreto y del estatuto. La primera era que respetara las leyes porque estaba en plena vigencia el estado de derecho, y que por tanto nunca los personajes de la dictadura le podían ganar un juicio a la Democracia, que es lo que sucedió en la normalización (el destacado del entrevistador). Le dimos la instrucción al Secretario de Asuntos Jurídicos para que controlara los actos administrativos y evitara que se cometieran ilegalidades. Por eso no se anularon las designaciones ya realizadas por el proceso, que habían otorgado derechos adquiridos, y que por lo tanto no podían revisarse en forma legal, 
tal como fue el caso del ex rector, Guillermo G. Gallo, lo que se sostuvo pese al reiterado reclamo de los estudiantes.(19)

El segundo consejo fue que les pidiera la renuncia a todos los funcionarios impuestos por la Dictadura que no fueran de carrera o simplemente los diera de baja y que inmediatamente -en el primer mes- designara a todos los colaboradores, porque si no iba a ver entorpecida la gestión durante meses. Se debían respetar las designaciones del personal de planta o de carrera. Los docentes interinos y los contratados -que depende de una decisión de la autoridad- que habían sido cómplices, podían ser dados de baja pero solo considerando la calidad de su designación interina, no por razones ideológicas. Se pudo reincorporar a varios cesanteados, docentes y no docentes desde 1976, porque en los considerandos de las resoluciones se citaban razones de "extrema peligrosidad" o conceptos persecutorios similares.

Y respecto de la ayuda recibida recuerdo que el Ministro Alconada, siempre respondió con gran espíritu republicano a todas las consultas que le realizamos ante las dudas, o el desconocimiento que podíamos tener de las cuestiones formales y legales.

Por supuesto que recibí una gran ayuda de los colaboradores, decanos y secretarios durante toda la gestión y fue gracias a ellos que se pudo normalizar la UNLP, en especial en la revisión de los concursos de la dictadura y en los más de mil concursos que se realizaron en menos de un año. Todavía estoy en deuda de agradecimiento para con ellos, ya que formamos un equipo coherente y firme durante toda la intervención. Pero debo destacar que el colaborador indispensable fue el Ing. Pablo O. Luchessi.(20)

GOQ: Volvemos al momento en que fue nombrado Rector Normalizador de la UNLP. ¿Cómo vivió ese momento?

RAP: Desde mediados de noviembre hasta que asumimos a fines de diciembre, la actividad fue vertiginosa, de tiempo completo, y también compleja por las decisiones que se tomaban. Teníamos gran convencimiento de lo que estábamos haciendo, muchas ideas y la fuerza necesaria para hacer el trabajo, así que vivimos ese mes con mucha euforia y excitación.

Para seleccionar a los decanos y secretarios, tendimos una red de consulta y recomendaciones con todos nuestros conocidos -personales- docentes, partidarios y de la Franja Morada, para constatar la calidad profesional y democrática de todas las personas propuestas. 
Con Pablo realizamos decenas de entrevistas, ya que no siempre aceptamos recomendaciones así no más, porque la decisión final era nuestra! Concertábamos las reuniones, para poder apreciar la personalidad y las ideas de cada persona en forma directa. De esa manera, el compromiso y el conocimiento fueron de persona a persona, sin intermediarios. Creo que esta manera de relacionarnos fue la que nos permitió formar un equipo coherente.

GOQ: ¿Pudo llevar adelante los objetivos que se habían fijado? ¿Piensa que la UNLP logró organizarse coherentemente con la letra de ley?

RAP: Creo que normalizamos la UNLP y que cumplimos con creces los objetivos que nos habían propuesto. Se designaron por concurso la mayoría de los cargos de profesores y se revisaron todos las designaciones del proceso -en total más de mil quinientos concursosreformamos el estatuto, descentralizamos la estructura de investigación de ciencia y tecnología, organizamos el ingreso directo con cursos de capacitación, aumentamos un 30\% la matrícula de los colegios y reformulamos el ingreso para garantizar la igualdad de oportunidad a toda la comunidad platense, con el sorteo de las vacantes.

También reformamos varios planes de estudio, aprobamos la nueva estructura de ordenanzas de concursos, de las elecciones y de juicio académico, creamos la Junta Ejecutiva y reincorporamos a docentes y no docentes expulsados por razones políticas. Hubieron otras tareas y proyectos que fracasaron, como por ejemplo los del traslado de Agronomía y Veterinaria, y el proyecto de departamentalización.

Se hizo mucho, y fíjese que casi todos los cambios que se hicieron aun hoy se mantienen presentes (más de treinta años después), aunque se hayan modificado algunos detalles. Dejamos una universidad con muchos problemas resueltos, muchos menos que con los que nos habíamos encontrado. Además con una experiencia, una cultura de autonomía, y un ejercicio de democracia interna que solo necesitaba la sencilla adecuación a la nueva legislación universitaria y al estatuto ya actualizado.

GOQ: ¿Si pudiera hacerlo, qué cosa modificaría de lo que se hizo durante el período de normalización?

RAP: De lo que hicimos creo que solo modificaría no haber vetado o anulado la forma de votación nominal en la Asamblea Universitaria para elegir al Presidente, que se decidió 
cuando se reformó el Estatuto en 1985, y que contó con mi oposición. Creo que esa forma de votar hace la elección menos independiente y libre que la del voto secreto.

En términos generales, creo que deberíamos haber realizado más cambios e innovaciones, porque la universidad es una entidad conservadora y prefiere mantener el statu quo más que analizar y cambiar las costumbres que pueden mejorar su funcionamiento.

Ya como pensamiento posterior, estimo que se debió y se debe ahora priorizar la docencia de grado y la formación profesional, mejorando su calidad y adecuando sus contenidos e innovando con las nuevas tecnologías educativas. En ese sentido se intentó desarrollar carreras intermedias con salida laboral, pero la idea fue rechazada, al igual que la idea de retomar el bachillerato nocturno en los colegios.

Creo que la Universidad, desde hace alrededor de dos décadas, cambió y adecuó la estructura reformista hasta convertirla en una máquina electoral y agencia de empleos al servicio de la formación de adherentes al populismo, educando con un equivocado relato político e histórico. Aún creemos en la filosofía política de la Reforma, con una Universidad autónoma, plural de ideas para la formación de ciudadanos en el espíritu de la democracia republicana de la Constitución. Una Universidad que sobre la base de esos principios podría retomar un impulso renovador, tal como se hizo en 1983, cuando refundamos la Universidad Nacional de La Plata.

GOQ: Muchas gracias.

Notas

(1) La literatura sobre la Reforma es muy amplia. Escritores reformistas produjeron una bibliografía que puede considerarse como clásica y al mismo tiempo como una fuente para el estudio de este movimiento. Por ejemplo: del Mazo, Gabriel. La Reforma Universitaria, La Plata, CEI, 1941; Ingenieros, José. La Universidad del porvenir, Buenos Aires, Ateneo, 1920; Palacios, Alfredo. La Universidad nueva, Buenos Aires, M. Gleizer, 1925; González, Julio V. La Revolución Universitaria, Buenos Aires, J. Menéndez Editor, 1922; Haya de la Torres, Víctor Raúl. La Reforma Universitaria, 1928; Mariátegui, José Carlos. La Reforma Universitaria, Lima, Amauta, 1928.

(2) Sobre las agrupaciones estudiantiles de las décadas del 50 y 60 en La Plata, se pueden consultar entre otros a Nava, Agustín. "Radicalización y politización del movimiento estudiantil: el caso platense durante la "Revolución Argentina". 1966-1972", en Revista del Programa de Investigaciones sobre Conflicto Social, Vol. 6 N 9, Enero 2013 a Junio 2013, pp. 93-120. Recuperado de

(25/11/2017): http://webiigg.sociales.uba.ar/conflictosocial/revista/09/08 A.Nava.pdf; y Pessacq, Raúl Adolfo. Notas sobre la Universidad de La Plata, de 1953 a 2017, Mimeo inédito, en proceso de edición.

(3) Esta entrevista se realizó el día 28/11/2017 en el domicilio del entrevistado a quien agradecemos su predisposición al diálogo. Se ha procurado intervenir lo menos posible en el 
texto. Se lo hace a los efectos de mejorar la edición escrita. El lector encontrará a pie de página, alguna información o nota aclaratoria sobre las cuestiones que se hablan y que ameriten hacerlo. Todo ello corre por cuenta del entrevistador.

(4) Fue creada por un grupo de militantes y adherentes a la Unión Cívica Radical en 1980. Además de los citados formaron parte de la misma -entre otros- Pablo Pinto; "Pincho" Orruma; Gustavo Calleja; Marcelo Marcó; Jorge A Lombardi, Omar Alfredo Iglesias; Angel Plastino; Alberto Fontán; Pablo O Luchessi; Raúl Alconada Sempé

(5) Participaron de la reunión, entre otros Manuel Sadosky; Carlos Abeledo; Jorge y Elba Roulet

(6) René Barbich fue dirigente estudiantil en la década del 50 en la Facultad de Ingeniería y trabajó en la Municipalidad de La Plata durante la presidencia de Arturo Illia. Federico Storani es Profesor en la UNLP, fue militante estudiantil, Diputado Nacional por la UCR, miembro de la Junta Coordinadora Nacional -línea interna de aquel partido-, Ministro del Interior de la Nación, etc. Andrés Ringuelet fue Ingeniero Agrónomo de la Universidad Nacional de La Plata, Profesor y antiguo militante reformista (datos proporcionados por el entrevistado).

(7) Federación Universitaria de La Plata

(8) Demo era Ingeniero Químico y Profesor de la Facultad de Ingeniería y De Lorenzo era Ingeniero Civil y fue entre otras cosas, Subsecretario de Obras Públicas de la Provincia de Buenos Aires entre 1984-88.

(9) La solicitada puede consultarse en el diario El Día, 31/05/1974

(10) Entre otras cosas, se les impidió deliberar en una Asamblea por ellos convocada. La noticia se encuentra en El Día, 02/06/1974

(11) Recuérdese que el 30 de octubre de 1983 había ganado las elecciones el candidato de la UCR, Raúl R. Alfonsín.

(12) Carlos Román Santiago Alconada Aramburú fue Ministro de Educación y Justicia entre 1983 y 1986.

(13) Tanto el Decreto 154 como el 228 se pueden consultar en Pessacq, Raúl A; Fernández Cortes, Silvina y Caorsi, Gabriela. La normalización de la Universidad Nacional de La Plata, La Plata, UNLP, 1987, pp. 1-3

(14) Alconada Aramburu, Carlos R. S. La República Democrática, obra del Radicalismo, Bs. As., UCR. 1983

(15) La denominada ley Avellaneda se puede consultar en https://www.unc.edu.ar/sobre-launc/nacionalización-y-ley-avellaneda Sobre el decreto 6403/55 entre otros: Requena, Pablo Manuel. "La larga vida del Reformismo", en Integración y Conocimiento, 1.6, 2017. Cabe recordar que por entonces, las plataformas políticas partidarias eran muy importantes. Poseían un diagnóstico de situación, las propuestas de gobierno y el sentido político de las mismas. Las plataformas de la UCR y del Partido justicialista de la campaña de 1983 tenían unas 20-25 páginas.

(16) En 1951 se creó Consejo Nacional de Investigaciones Técnicas y Científicas, antecedente que dio lugar a la creación del Consejo Nacional de Investigaciones Científicas y Técnicas (CONICET). Fue fundado por Bernardo Alberto Houssay el 5 de febrero de 1958. Los datos en www.conicet.gov.ar/los-57-anos-del-conicet/ La CIC es la Comisión de Investigaciones Científicas de la Provincia de Buenos Aires. Su ley orgánica se origina en un Decreto Ley, el 7385/68. Datos en http://www.cic.gba.gob.ar/?page_id=891

(17) La "costumbre política" era que los interventores actuaran sin controles y estatutos, razón por la cual las políticas se ejecutaban con absoluta discrecionalidad.

(18) Para obtener un contexto histórico narrado por testigos de época véase a Delorenzo, Juan Carlos y Pessacq, Raúl Adolfo. Política y Universidad de 1945-2017. Una historia alternativa, La Plata, Grafikar, 2015, pp. 71-87

(19) El Dr. Guillermo Gallo había alcanzado el grado de Capitán Veterinario, es decir que era militar. Había recibido el título de Doctor en Medicina Veterinaria en la Facultad de Ciencias 
Veterinarias de la Universidad Nacional de La Plata en el año 1950, y fue Profesor de esa casa. Fue rector de la UNLP durante todos los años de la dictadura (años 1976 a 1983)

(20) El Ingeniero Pablo Luchessi, fue Secretario General de la Universidad durante la intervención y, entre otras cosas, fue durante los años 90 Diputado Provincial y Presidente de la Junta Central de la UCR. Para consultar la lista de Decanos y colaboradores ver Pessacq, Raúl A; Fernández Cortes, Silvina y Caorsi, Gabriela, Op. Cit.

Recibido: octubre de 2017.

Aprobado: diciembre de 2017.

\section{Para citar este trabajo}

Quinteros, G. “A cien años de la Reforma Universitaria: Entrevista a Raúl A. Pessacq, un reformista para construir la Universidad de la democracia" en Cuadernos de H Ideas [En línea], vol. 11, nº 11, diciembre 2017, consultado...; URL: http://perio.unlp.edu.ar/ojs/index.php/cps/article/view/4540 